\title{
Speed-accuracy tradeoff in choice reaction time: Within conditions, between conditions, and between subjects
}

\author{
G. ROBERT GRICE and V. ALAN SPIKER \\ University of New Mexico, Albuquerque, New Mexico 87131
}

\begin{abstract}
A quantitative theory of choice reaction time (CRT), developed in the context of accurate performance was successfully applied to a speeded experiment. With estimation of a single parameter, mean criterion level, the theory described the reaction time (RT) distributions for correct responses and errors and the details of the speed-accuracy tradeoff. The form of the tradeoff within experiments is not invariant with a shift of criterion when described by conditional error probabilities. However, a new tradeoff function which is invariant under this condition is presented. Descriptions of individual performance and of individual differences are also provided. Error rates are determined primarily by the amount of criterion variability and the ability to inhibit short-latency errors. Slower subjects with relatively high criterion levels tend to make the most errors. Previous presentations of the theory are extended by a more explicit analysis of the implications of the theory for the speed-accuracy tradeoff.
\end{abstract}

Application of the basic principles of variable criterion theory to choice reaction time (CRT) has led to the formulation of a more complete theory of CRT to simple auditory stimuli (Grice, Nullmeyer, \& Spiker, 1977; Grice, Spiker, \& Nullmeyer, 1979). The intent of this theory is to identify the underlying processes leading to response evocation, and to provide quantitative descriptions of the growth of these processes in the time following stimulus onset. One of the properties of the approach is that it provides a description of the tradeoff between speed and accuracy, and a theoretical account of its dynamics.

The speed-accuracy tradeoff is typically investigated in one of two ways-between conditions or within conditions. In the between-conditions approach, the speed-accuracy emphasis is experimentally manipulated across conditions and a measure of accuracy is plotted against a measure of central tendency of RT for each condition. Accuracy typically increases with time, and such relations have been termed by Wood and Jennings (1976) "speed-accuracy tradeoff functions" (SATFs). In the within-conditions approach, responses are grouped according to latency within a condition, and a measure of accuracy is plotted against the latencies of the groups. These relations also indicate that accuracy increases with increasing RT within the range of the tradeoff. They have been called by Wood and Jennings "con-

This research was supported by United States Public Health Service Grant MH 16400 from the National Institute of Mental Health. Requests for reprints should be sent to G. Robert Grice, Department of Psychology, University of New Mexico, Albuquerque, New Mexico 87131. ditional accuracy functions"' (CAFs) i.e., they are based on the conditional probability that a response is correct, given that it occurs at a particular time. ${ }^{1}$ The form of a CAF tends not to be the same as that of an SATF. In this connection, Wood and Jennings raised the interesting question as to whether the CAF is invariant over between-conditions changes in the criterion, and present convincing evidence that it is not. The variable criterion theory of CRT predicts this lack of invariance and provides a theoretical account of why it is so. A third possible source of the tradeoff might be based upon individual differences and the reasonable hypothesis that fast subjects should make more errors than slow ones. While the individual differences analysis provided by Grice et al. (1979) indicated no evidence for this, this possibility is investigated further in this paper.

The paper by Grice et al (1979) was an analysis of stimulus similarity in terms of variable criterion theory, and it presented parameters of the theory's functions for experiments involving three degrees of similarity. The experiments were conducted under accuracy instructions. The present research utilizes the theory for the intermediate level of similarity (tones of 1,000 and $1,100 \mathrm{~Hz}$ ) to predict performance in a new experiment conducted under speeded conditions. Features of the speed-accuracy tradeoff both within and between conditions are examined in terms of the theory, and patterns of individual differences are also analyzed. Since the theory is applied separately to each subject, the new experiment may be viewed as 25 replications, under speeded conditions, of the original accuracy experiment. The group data 
of the two experiments are also analyzed and compared.

The theory is as described by Grice et al. (1979). Following stimulus onset, the excitatory strengths of the two responses grow as deterministic functions of time. When the excitatory strength of either response reaches the value of its criterion on that trial, that response occurs and the trial is terminated. The criteria for the two responses are random variables, normally distributed over trials. The two criterion distributions are independent, and, in the present application, have equal means and standard deviations. Excitatory strength of the correct response is described as follows:

$$
\mathrm{E}_{\mathrm{c}}(\mathrm{t})=(\mathrm{V}(\mathrm{t})+\mathrm{A}(\mathrm{t})-\overline{\mathrm{C}}) / \mathrm{o}
$$

where $E_{c}(t)$ is the expected value of excitatory strength at time $t, V(t)$ is the value of the sensory detection component at $t, A(t)$ is associative strength to the correct stimulus at $t, \bar{C}$ is the mean of the criterion distribution, and $\sigma$ is its standard deviation. Both $\mathrm{V}$ and $\mathrm{A}$ are monotone increasing functions of time. Excitatory strength for the incorrect response is given by:

$$
E_{e}(t)=\left[E_{c}(t)-I(t)-A_{D}(t)-\bar{C}\right] / \sigma,
$$

where $E_{e}(t)$ is excitatory strength at time $t, I$ is associative inhibition at $t$, and $A_{D}$ is associative discrimination at $t$. Both $I$ and $A_{D}$ are also monotone increasing functions of time. The specific functions applicable to the 1,000 - vs, $1,100-\mathrm{Hz}$ experiments are as follows:

$$
\begin{aligned}
V(t) & =.378-17.549 \mathrm{e}^{-.00758 \mathrm{t}}, \\
\mathrm{A}(\mathrm{t}) & =2.050\left(1.144 \times 10^{-15}\right) .9930^{\mathrm{t}} \\
\mathrm{I}(\mathrm{t}) & =1.909\left(9.621 \times 10^{-9}\right) .9891^{\mathrm{t}} \\
\mathrm{A}_{\mathrm{D}}(\mathrm{t}) & =1.480\left(6.621 \times 10^{-41}\right) .9914^{\mathrm{t}}
\end{aligned}
$$

with $t$ in milliseconds. ${ }^{2}$ Full functions for the growth of $E$ are obtained by inserting these functions in Equations 1 and 2 . On the scale of the original accuracy experiment, the values of $\bar{C}$ and $\sigma$ are zero and one. Growth of the cumulative theoretical probabilities, $F_{c}(t)$ and $F_{e}(t)$, of the correct and incorrect responses reaching criterion is given by the integral of the unit normal function from minus infinity to $E(t)$ at all values of $E(t)$.

A trial in CRT may be considered as a "race" between the growth of the excitatory strengths of the correct and incorrect responses. The first to reach its criterion is the one which occurs on the trial. Be- cause of the limitation of one response per trial, the distributions of overt correct responses and errors are determined by the theoretical probabilities of reaching criterion, but are not equal to them except in errorless performance. The relations derived by Grice et al (1979) are as follows:

$$
\Delta P_{c}(t)=\Delta F_{c}(t)\left[1-F_{e}(t)-.5 \Delta F_{c}(t) \Delta F_{e}(t)\right]
$$

and

$$
\Delta \mathrm{P}_{\mathrm{e}}(\mathrm{t})=\Delta \mathrm{F}_{\mathrm{e}}(\mathrm{t})\left[1-\mathrm{F}_{\mathrm{c}}(\mathrm{t})-.5 \Delta \mathrm{F}_{\mathrm{c}}(\mathrm{t}) \Delta \mathrm{F}_{\mathrm{e}}(\mathrm{t})\right],
$$

where $\Delta \mathrm{P}_{\mathrm{c}}(\mathrm{t})$ and $\Delta \mathrm{P}_{\mathrm{e}}(\mathrm{t})$ are the predicted probabilities of correct responses and errors in a brief interval, $\Delta \mathrm{t}$, beginning at $\mathrm{t} ; \Delta \mathrm{F}_{\mathrm{c}}(\mathrm{t})$ and $\Delta \mathrm{F}_{\mathrm{e}}(\mathrm{t})$ are the theoretical probabilities of each response reaching criterion within $\Delta t . \Delta P_{c}$ and $\Delta P_{e}$ are computed for each $\Delta t$ and summed to provide the full cumulative distributions for correct responses and errors. It is also frequently necessary to estimate the underlying theoretical functions $F_{c}$ and $F_{e}$ from data in the form of obtained distributions of correct responses and errors. This is accomplished by close approximations of the inverse of Equations 7 and 8.

$$
\Delta \mathrm{F}_{\mathrm{c}}(\mathrm{t})=\frac{\Delta \mathrm{P}_{\mathrm{c}}(\mathrm{t})}{1-\mathrm{F}_{\mathrm{e}}(\mathrm{t})}+\frac{.5\left[\Delta \mathrm{P}_{\mathrm{c}}(\mathrm{t}) / 1-\mathrm{F}_{\mathrm{e}}(\mathrm{t})\right]\left[\Delta \mathrm{P}_{\mathrm{e}}(\mathrm{t}) / 1-\mathrm{F}_{\mathrm{c}}(\mathrm{t})\right]}{1-\mathrm{F}_{\mathrm{e}}(\mathrm{t})}
$$

and

$$
\Delta \mathrm{F}_{\mathrm{e}}(\mathrm{t})=\frac{\Delta \mathrm{P}_{\mathrm{e}}(\mathrm{t})}{1-\mathrm{F}_{\mathrm{c}}(\mathrm{t})}+\frac{.5\left[\Delta \mathrm{P}_{\mathrm{c}}(\mathrm{t}) / 1-\mathrm{F}_{\mathrm{e}}(\mathrm{t})\right]\left[\Delta \mathrm{P}_{\mathrm{e}}(\mathrm{t}) / 1-\mathrm{F}_{\mathrm{c}}(\mathrm{t})\right]}{1-\mathrm{F}_{\mathrm{c}}(\mathrm{t})}
$$

where $\Delta P_{c}(t)$ and $\Delta P_{e}(t)$ are the obtained proportions of correct responses and errors within the brief interval, $\Delta$ t. $\Delta \mathrm{F}_{\mathrm{c}}(\mathrm{t})$ and $\Delta \mathrm{F}_{\mathrm{e}}(\mathrm{t})$ are computed and successively summed beginning with the earliest nonzero interval. This yialds the complete functions for $F_{c}$ and $\mathrm{F}_{\mathrm{e}}$. It may also be noted that the normal deviates of these values provide estimates for the growth of $E_{c}$ and $E_{e}$ in scale units.

The basic hypothesis of the present research is that Equations 3-6 will remain invariant for all subjects under an increase in speed requirements. If this is true, the theory should describe the distributions of the individual subjects and of the group, as well as the nature of the speed-accuracy tradeoff. The only parameters free to vary are the three previously shown to be subject to individual differences, one of which should also be influenced by the experimental manipulation (Grice et al., 1977; Grice et al., 1979). These three variables are $\bar{C}, \sigma$, and $W_{I} . W_{I}$ is a weighting factor applied to the inhibition function, 
Equation 5, only. It has been shown to be a significant individual-difference variable related to error rate. The main influence of the speeding should be a decrease in $\bar{C}$, the mean level of the criterion distribution. Possible influences on the other two variables cannot be ruled out, however, and this question is under investigation.

\section{METHOD}

Details of the method have been presented previously (Grice et al., 1977; Grice et al., 1979). Responses were made to two keys operated with the two index fingers. The stimuli were $100-\mathrm{dB}$ SPL tones of 1,000 and $1,100 \mathrm{~Hz}$, the higher tone being associated with the right hand. In the present experiment, subjects were instructed to meet a deadline which was based on the third quartile of the accuracy experiment. Immediate visual feedback was given for errors and for responses slower than the deadline. There were 24 practice trials followed by 200 trials in which the two stimuli were presented equally often in an irregular order. Subjects in the deadline experiment were 25 women from an introductory psychology course, and they received course credit for participation. There were 22 subjects in the previously reported accuracy experiment.

\section{RESULTS AND THEORETICAL ANALYSIS}

\section{Summary Data}

A summary of the RT and error rate data for the two experiments is presented in Table 1 . Here are presented the means and standard deviations of the subject median RTs for correct responses and errors in each experiment. The RTs are typical of those obtained in our laboratory from similar subjects, but are a little longer than those obtained with practiced subjects. Mean and SD error rates are also included. The data indicate a typical between-conditions speed-accuracy tradeoff. RTs are significantly faster in the deadline experiment for both correct responses and errors. For correct responses, $\mathrm{t}(45)=7.80$ $\mathrm{p}<.001$; and for errors, $\mathrm{t}(44)=5.37, \mathrm{p}<.001$. Error rate is significantly greater in the deadline experiment, $t(45)=3.56, \mathrm{p}<.001$. Also in both experiments, we obtained the typical results that errors are significantly faster than correct responses. For the accuracy experiment, $t(20)=5.64, p<$ .001 ; and for the deadline experiment, $t(24)=5.81$, $\mathrm{p}<.001$.

\section{Prediction of the Individual Subject Distributions}

Applicability of the theory to the subjects of the deadline experiment is evaluated by obtaining, for each subject, estimates of the three individual difference parameters in terms of the theory. This permits the calculation of predicted distributions for both correct responses and errors which may then be compared to the obtained distributions. Computations were essentially those described by Grice et al. (1977) and Grice et al (1979), but with minor improvements. ${ }^{3}$
Table 1

Means and Standard Deviations of Subject Median RTs in Milliseconds and of Subject Error Rates

\begin{tabular}{|c|c|c|c|c|}
\hline & \multicolumn{2}{|c|}{ Accuracy } & \multicolumn{2}{|c|}{ Deadline } \\
\hline & Mean & SD & Mean & SD \\
\hline Correct RT & 427 & 33 & 363 & 23 \\
\hline Error RT & 384 & 44 & 321 & 36 \\
\hline Error Rate & .053 & .034 & .115 & .075 \\
\hline
\end{tabular}

The theoretical analysis of the deadline experiment was limited to $600 \mathrm{msec}$, which includes $98.3 \%$ of all responses. Subject parameters and distributions for the accuracy experiment were presented by Grice et al. (1979). However, for comparison here, all have been recomputed with methods identical to those used for the deadline experiment. Differences from the original results were negligible.

Cumulative distributions of correct responses and errors predicted from the theory provided good fits to the obtained distributions of the 25 subjects. The proportion of variance in the data points of both correct and error distributions accounted for, within distributions, varied from a low of .984 to .999 . The mean proportion was .993 and the median was .994. Graphs of the predicted and obtained distributions, of eight representative subjects are presented in Figure 1. The identifying numbers to the left of each figure indicates the rank order of goodness of fit defined by proportion of variance accounted for. Thus, the subject in the upper left corner represents the poorest fit, and the subject in the lower right is the fourth best of the 25 . Numbers to the right indicate, from top to bottom, the subject parameters $\bar{C}, \sigma$, and $\mathrm{W}_{\mathrm{I}}$. Some of the dynamics of the theory may be observed here. For example, Subject 19, who had the highest error rate, had a moderate criterion level, but the higher error rate is produced by very high criterion variability and low $\mathrm{W}_{\mathrm{I}}$. An interesting comparison is between Subjects 23 and 13, who have equal criterion levels and comparable $\mathrm{W}_{\mathrm{I}}$. The higher error rate of subject 23 is attributable entirely to higher criterion variability. Subjects 7 and 15 have very low criterion levels, but achieve low error rates as a result of low criterion variability and high levels of the kind of discriminative ability measured by $\mathrm{W}_{\mathrm{I}}$.

Fits of the recalculated distributions for the accuracy experiment were excellent. The pooled proportion of variance accounted for in the two distributions varied from .991 to seven subjects with .999 or higher. The mean and median proportions were .997. Graphs of the distributions of representative subjects were presented by Grice et al. (1979).

One basis for examining the nature of the between conditions tradeoff is a comparison of the three individual-difference parameters. Means and SDs of 


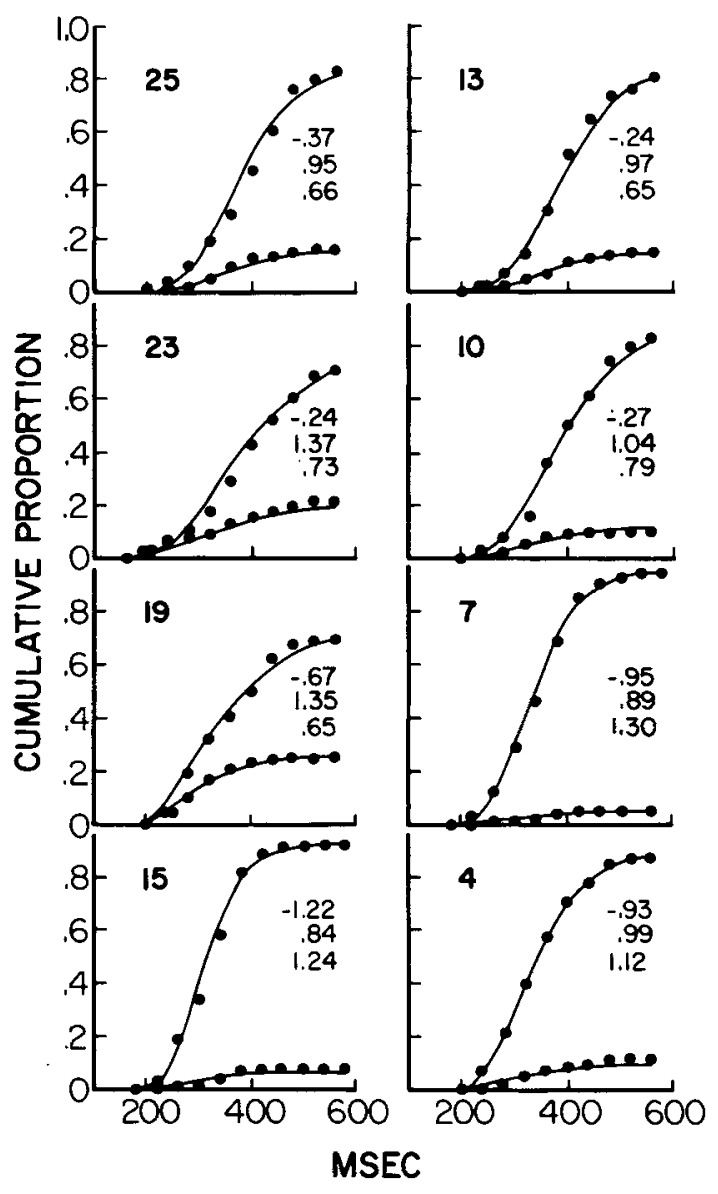

Figure 1. Predicted and obtained cumulative distributions for eight subjects in the deadline experiment. Upper functions are for correct responses and lower functions are for errors. Numbers to the left of each figure indicate the rank order of goodness of fit of the two distributions in the group of 25 subjects. Numbers to the right indicate, from top to bottom, the values of $\overline{\mathbf{C}}, \sigma$, and $\mathbf{w}_{\mathbf{I}}$.

these variables for the two experiments are presented in Table 2 . The only variable differing significantly between the experiments is the mean criterion level, $\overline{\mathrm{C}} ; \mathrm{t}(45)=7.82, \mathrm{p}<. .001$. Differences between the means of $\sigma$ and $W_{I}$ are small and not significant. For $\sigma, \mathrm{t}(45)=.80$, n.s.; and for $\mathrm{W}_{\mathrm{I}}, \mathrm{t}(45)=.51$, n.s. What this means is that the basis of the tradeoff, in this instance, was almost entirely a difference in mean criterion level. It will be of interest to note how well other features of the data support this interpretation.

\section{Prediction of the Group Distributions}

As shown in the earlier papers, the theory applies to the combined group distributions of correct responses and errors as well as those of individual subjects if the group criterion distribution is approximately normal. This is a considerable convenience here, since the speed-accuracy tradeoff can be examined better with the more reliable group data. The parameters of the group may be obtained directly from the individual subject values without any estimates from the group distributions themselves. The mean of the group criterion distribution is the mean of the subject means. Its variance is the sum of the variance of the subject criterion means and the pooled variance within subjects. An appropriate estimate of $W_{I}$ is the mean of the subject values. The theoretical equations are transformed to the group scales by these parameters, and group distributions are calculated in the same manner as for individual subjects (Steps 7 to 9 of the preceding note). Estimates of the form of the two criterion distributions indicate that they closely approximate normality. ${ }^{4}$

The calculated and obtained cumulative distributions of correct responses and errors for the accuracy experiment are presented in the upper panel of Figure 2. Data points are at $20-\mathrm{msec}$ intervals. Obviously the fit is excellent. The proportion of vari-

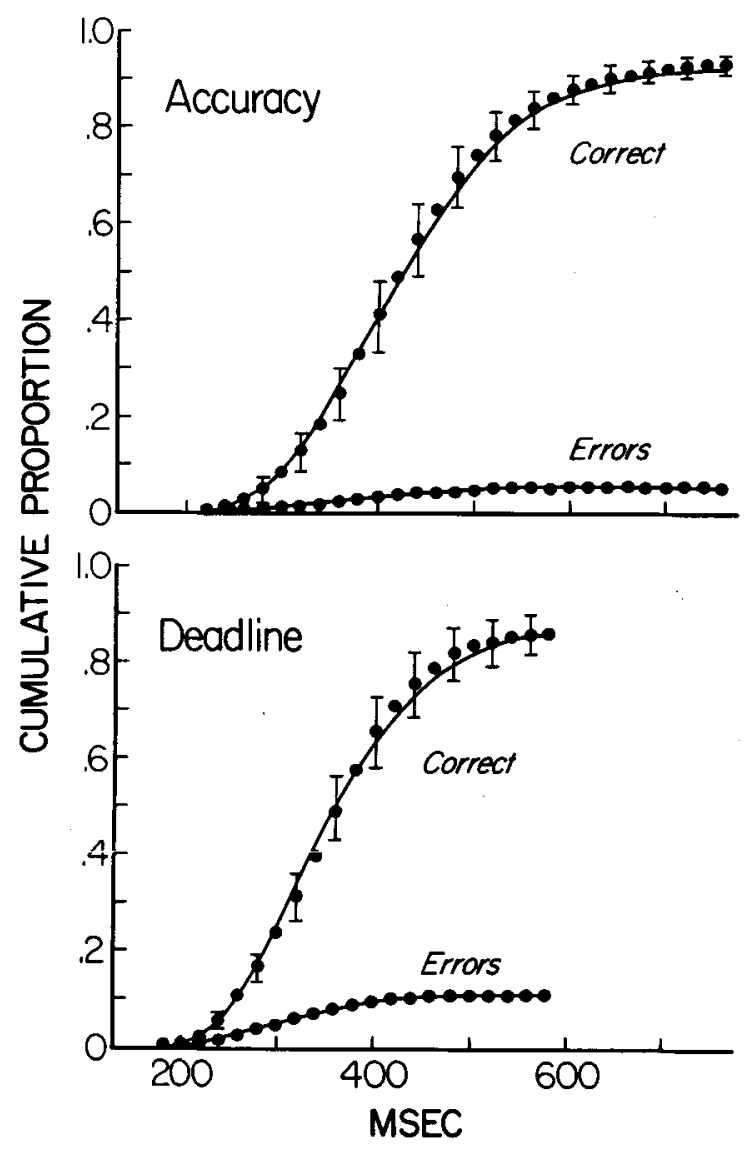

Figure 2. Predicted and obtained cumulative group distributions for the accuracy and deadline experiments. Vertical lines associated with data points of the correct distributions indicate the range of \pm 2.5 times the standard error of the mean of the subject proportions. 
ance of the correct and error data points accounted for, within distributions, is .999 . Of course, the chief interest here is in whether the theory predicts the distributions of the new deadline experiment. This prediction was obtained in two ways. One method, the same as for the accuracy condition, is based on the three individual-difference parameters. The other method uses only the mean criterion levelthe only variable for which a prediction was made. In this procedure, the equations for the deadline experiment are the same as for accuracy except that difference between the mean criterion levels, as indicated in Table 2, is subtracted from the value of $\bar{C}$. The results of the two procedures were about the same. While the calculated distributions for the deadline experiment in Figure 2 are drawn from the threeparameter solution, the two sets of functions are scarcely discernible. The single-parameter solution was slightly superior for the correct distribution and slightly inferior for the error distribution. The proportion of variance of the data points accounted for in the two distributions was .997 for both solutions. Thus, the theory describes both distributions of the speeded performance on the basis of a single parameter. Since the parameter, $\bar{C}$, was estimated from the correct functions only, the prediction of the error distribution was parameter free.

There is another kind of analysis which confirms and further describes the single-parameter difference between the two experiments. This is in terms of the functions for the growth of excitatory strength as obtained directly from the empirical data. From Equation 1, it is seen that any point on a correct function is an additive combination of an increasing function $[V(t)+A(t)]$ and a constant $(\bar{C})$. If the functions for the two experiments are the same, but the values of $\bar{C}$ differ, then when plotted the function should be parallel. The same reasoning applies to the functions for errors. Also, since the values of $\overline{\mathrm{C}}$ are the same for correct and errors, the distances between the two pairs of parallel function should be equal. That these predictions are very closely approximated by the data is shown in Figure 3. These functions are derived directly from the group distributions by a two-stage transformation. First, the estimated probabilities of reaching criterion were obtained by Equations 9 and 10, and these estimates were further transformed by the inverse normal function. The mean distance between the correct functions is .702 , and that between the error functions is .726. Figure 3 is similar in form and meaning to figures commonly presented by Anderson (1974) to illustrate additive models in his applications of functional measurement to integration theory.

\section{The Speed-Accuracy Tradeoff}

\section{Within and Between Conditions}

We now present descriptions of the speed-accuracy tradeoff within each of the two experiments and with
Table 2

Means and Standard Deviations of the Three Theoretical Individual Difference Parameters

\begin{tabular}{llllll}
\hline & \multicolumn{2}{c}{ Accuracy } & & \multicolumn{2}{c}{ Deadline } \\
\cline { 2 - 3 } \cline { 5 - 6 } & Mean & SD & & Mean & SD \\
\hline$\overline{\mathrm{C}}$ & -.006 & .312 & & -.737 & .327 \\
$\sigma$ & .973 & .202 & & 1.019 & .192 \\
$\mathrm{~W}_{\mathrm{I}}$ & .994 & .282 & & 1.040 & .317 \\
\hline
\end{tabular}

a comparison of the two experiments. These descriptions are in terms of the complement of CAFs, conditional error functions (CEFs). We present these in alternative ways. The most common way is to rank all responses by $R T$ and divide them into equal groups of increasing RT. Here we divided the responses into successive fifths and plotted the probability of an error against the median RT of the group. This appears in the upper left of Figure 4. Alternatively, in the upper right, we plot the probability of an error within equal time intervals. Points are at the upper limits of 100-msec intervals. In the lower figures, the functions are presented cumulatively, i.e., the conditional probability of an error given that a response has occurred by a given time. It makes sense to look at the data this way when one considers information accrual following stimulus onset to be a cumulative process. The lines in Figure 4 connect points calculated from the predicted distributions.

All of the representations indicate basically the same picture. Conditional error probability decreases as a negatively accelerated function of time since stimulus onset. It is also clear that the CEFs are not invariant over the two experiments. Thus, both the findings of Wood and Jennings (1976) and the prediction of the theory are confirmed. It is not true

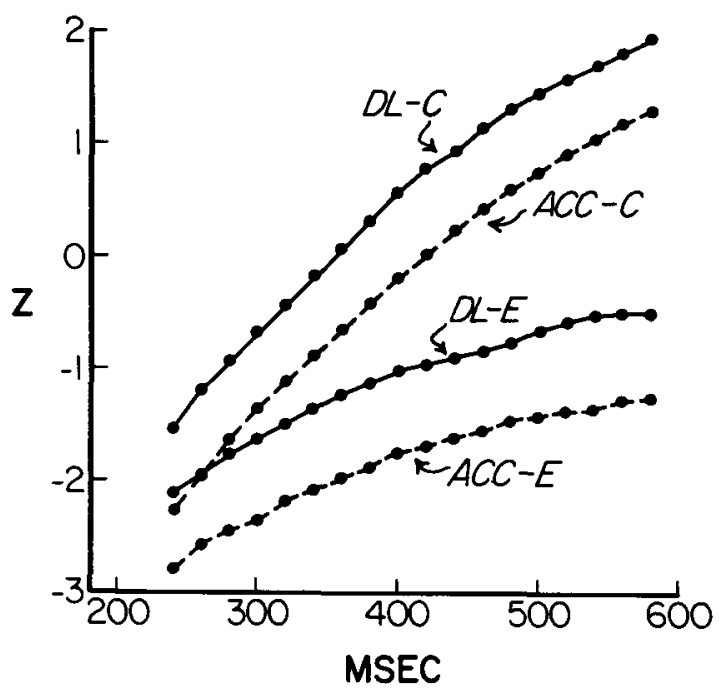

Figure 3. Empirically derived functions for the growth of excitatory strength $(Z)$ for the deadline (DL) and accuracy $(A C C)$ experiments for correct responses (C) and errors (E). See text for details. 

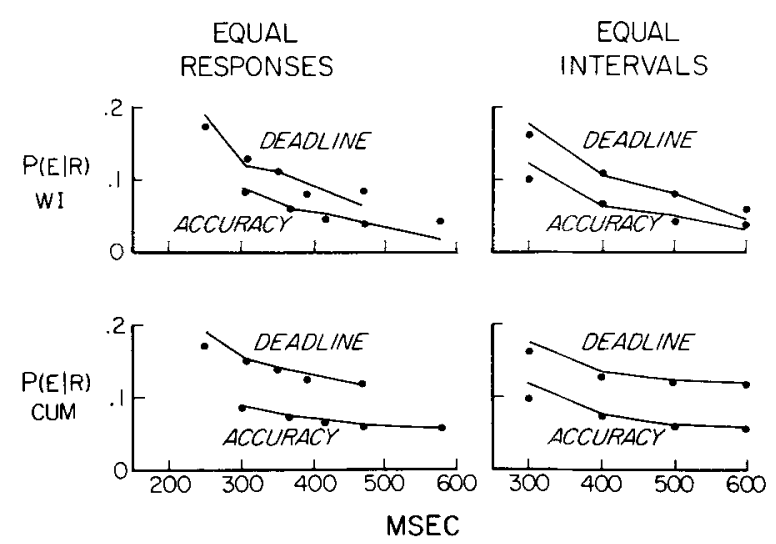

Figure 4. Conditional error functions (CEFs) for the deadline and accuracy experiments. The upper functions given the conditional probabilities of errors given that a response occurs within (WI) successive intervals. The lower functions given the cumulative (CUM) conditional probabilities. On the left, intervals are defined by the occurrence of equal numbers of responses. On the left, they are equal intervals of $\mathbf{1 0 0}$ msec.

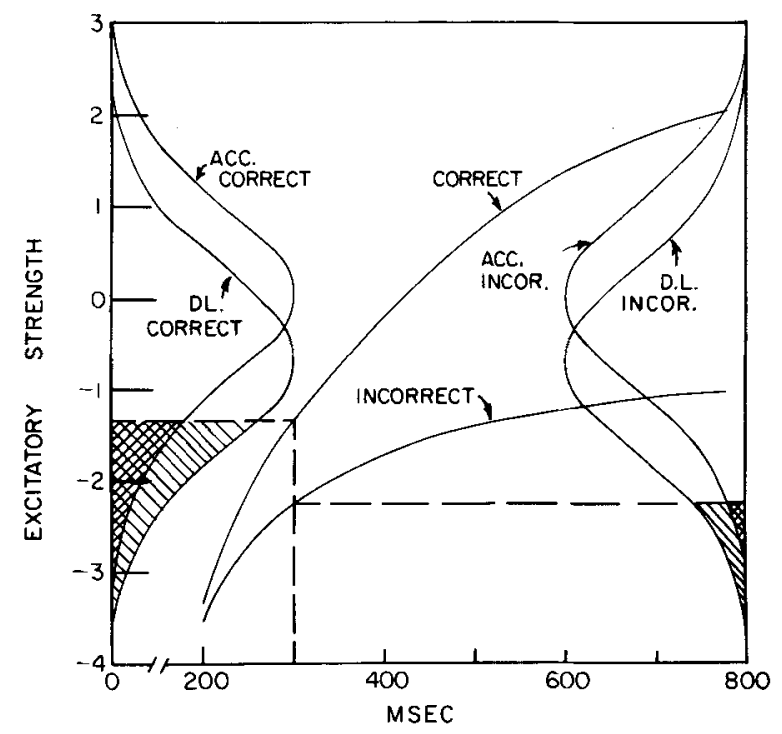

Figure 5. Graphic illustration of the theory in which the two experiments differ only in mean criterion level. The monotone, increasing functions represent the calculated growth of excitatory strength for the correct and incorrect responses. Normal functions at the left indicate the criterion distributions for the correct response for the accuracy $(A C C)$ and deadline (DL) experiments. Criterion distributions for the incorrect response are at the right. Cross-hatched areas indicate the theoretical probabilities of each response, in each experiment, reaching criterion by 300 msec.

that a response occurring at a particular time has a fixed probability of being an error that is independent of the mean criterion level of the condition. At all times, conditional error probabilities are higher for the deadline experiment.

The nature of this prediction is illustrated in a graphic representation of the theory presented in Figure 5. This figure is constructed according to the condition that the experiments differ only in the single parameter, $\bar{C}$. The origin is at the mean of the accuracy criterion distribution, and the unit of excitatory strength is the standard deviation of that distribution. Functions indicating the growth of correct, $E_{c}(t)$, and incorrect, $E_{e}(t)$, excitatory strength are computed from Equations 1 and 2, including the component functions of Equations 3-6. Normal criterion distributions for the correct response for each experiments are indicated along the ordinate at the left. They are of equal standard deviations, and, in units of the accuracy group criterion distribution, the difference between their means is $\mathbf{. 7 0}$. Identical distributions of the criterion for the incorrect response are indicated at the right. Predictions of the theory are illustrated at $300 \mathrm{msec}$. The vertical line at this point indicates the ordinates of the two excitatory strength functions. Horizontal lines are extended to the distributions at these points. Areas below these lines indicate the theoretical probabilities of reaching criterion by $300 \mathrm{msec}$ for each response in each experiment. The basis of the dependence of conditional error rate on mean criterion level may be seen from these values. For the accuracy experiment, these probabilities are .091 for correct responses and .012 for errors. The corresponding probabilities for the deadline experiment are .274 and .064 . In terms of these theoretical values, the conditional error probabilities are .117 for accuracy and .189 for deadline. However, in order to obtain these predictions in terms of predicted response, the race property of the theory must be taken into consideration through the application of Equations 7 and 8. When this is done, the predicted response probabilities are .090 and .012 for accuracy and .264 and .056 for deadline. Thus, the predicted conditional error rates for the interval under $300 \mathrm{msec}$ are .118 for accuracy and .175 for deadline. As may be seen from Figure 4, this dependence upon mean criterion level exists throughout the range of the present data.

The present theory provides a function, descriptive of the within-condition speed-accuracy tradeoff, which is, in principle, invariant over between-condition differences in criterion level only. This is readily seen in Figure 5, where the functions for excitatory strength apply to both experiments. The difference between the two functions, $E_{c}(t)-E_{e}(t)$, measures, in scale units, the increase with time of discriminative accuracy. This measure is equally applicable to the two experiments. In terms of Equations 1 and 2, this value is also equal to $I(t)+A_{D}(t)$, the sum of the two discriminative terms of the theory. Such functions were presented by Grice et al. (1979) as a way of describing the effects of stimulus similarity. As a description of empirical data, the function consists of the difference between the correct and error transformations presented in Figure 3. An appropriate name for the function would be "variable criterion difference function" or "variable criterion 
discrimination function" (VCDF). These functions for the accuracy and deadline experiments are presented in Figure 6. For legibility, the points are plotted only at alternate 20 -msec intervals. The line is calculated from Equations 1 and 2 on the accuracy group scale. As expected, the two functions are nearly the same. For all points, the proportions of variance accounted for by the calculated curve are .996 for accuracy and .987 for deadline. Thus, sampling error and the small differences in the parameters, $\sigma$ and $W_{I}$, indicated in Table 2 account for only $1.3 \%$ of the variance for the deadline experiment.

\section{Individual Differences}

Individual differences are examined by means of a multiple regression analysis applied to the relation between error rate (ER) and the three individual difference variables of the theory. The intercorrelations of these variables for the two experiments are presented in Table 3. The multiple correlations (R) for predicting error rate from the three theoretical variables are .896 for the accuracy experiment and .983 for the deadline experiment. Presumably, the higher correlation for the deadline experiment reflects the substantially greater variance of ER. The multiple regression equations, in standard score form, for the prediction of ER are, for the accuracy experiment,

$$
\mathrm{Z}_{\mathrm{ER}}=-.483 \mathrm{Z}_{\overline{\mathrm{C}}}+1.010 \mathrm{Z}_{\mathrm{o}}-1.193 \mathrm{Z}_{\mathrm{W}_{\mathrm{l}}},
$$

and for the deadline experiment,

$$
\mathrm{Z}_{\mathrm{ER}}=-.415 \mathrm{Z}_{\overline{\mathrm{C}}}+.604 \mathrm{Z}_{\sigma}-.928 \mathrm{Z}_{\mathrm{W}_{\mathrm{I}}} .
$$

The sign of each regression weight is in the direction of the obvious relationship predicted by the theory. In both experiments, the largest weight is for $\mathrm{W}_{\mathrm{I}}$.

In Table 4, we present the measure of importance or usefulness (U) which is the reduction in $R^{2}$ when a variable is omitted from the prediction. The values of $F$ for each are also included in Table 4. Each variable contributes significantly to the prediction in both experiments, although the contribution of $\bar{C}$ is small. As defined by $U$, the most important contributor in both experiments is $o$. Criterion variability has not been widely recognized in the literature as a major cause of errors. The prediction of the present theory is quite clear. For example, with respect to Figure 5, it is readily seen that an increase in criterion variability would decrease the distance between the correct and error growth functions in $o$ units and decrease the slope of the VCDF. The area of the criterion distribution below the error function would also be increased throughout the function. Since individual differences in criterion variability are substantial, it is not surprising that this variable

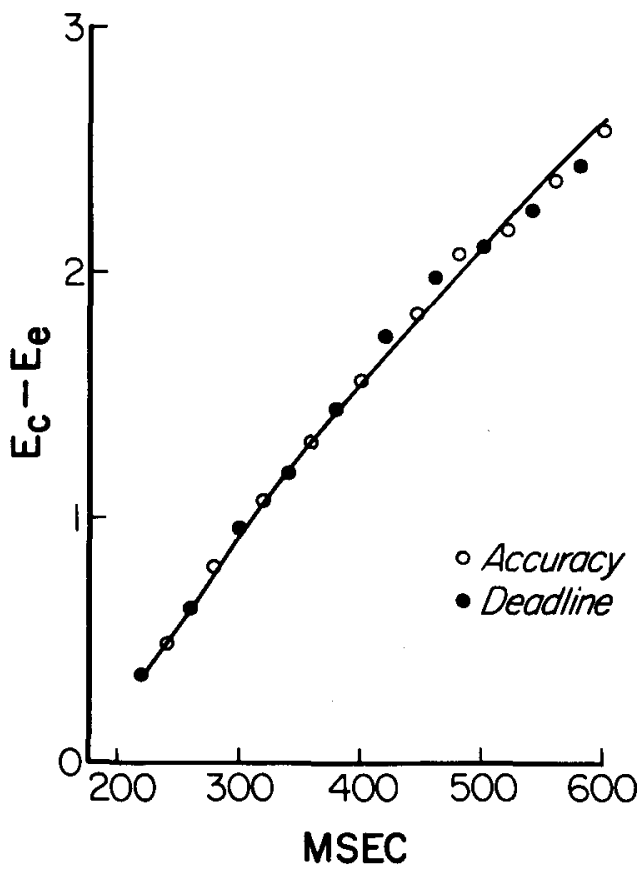

Figure 6. Variable criterion discrimination functions (VCDFs) for the two experiments. The smooth curve represents the difference between correct and error excitatory strength $\left(E_{c}-E_{e}\right)$ calculated from the theory for the accuracy group data. Data points are the corresponding differences between the empirical functions presented in Figure 3.

should be a major determinant of differences in error rate. This finding replicates that of Grice et al. (1979) in two other experiments.

Table 3

Intercorrelations of Error Rate (ER) and the Three Theoretical Individual Difference Variables

\begin{tabular}{cccccccc}
\hline & \multicolumn{3}{c}{ Accuracy Experiment } & & \multicolumn{3}{c}{ Deadline Experiment } \\
\cline { 2 - 4 } \cline { 6 - 8 } & $\overline{\mathrm{C}}$ & $\sigma$ & $\mathrm{W}_{\mathrm{I}}$ & & $\overline{\mathrm{C}}$ & $\sigma$ & $\mathrm{W}_{\mathrm{I}}$ \\
\hline $\mathrm{ER}$ & .162 & .496 & -.319 & .625 & .792 & -.805 \\
$\overline{\mathrm{C}}$ & & -.218 & -.725 & & .407 & -.856 \\
$\sigma$ & & & .519 & & & -.385 \\
\hline
\end{tabular}

Table 4

Values of $F$ and Measures of Importance (U) for Each Theoretical Variable Contributing to the Multiple Regression Equations Predicting Error Rate

\begin{tabular}{lccccc}
\hline & \multicolumn{2}{c}{$\begin{array}{c}\text { Accuracy } \\
\text { Experiment } \\
(\mathrm{df}=1,18)\end{array}$} & & \multicolumn{2}{c}{$\begin{array}{c}\text { Deadline } \\
\text { Experiment } \\
(\mathrm{df}=1,21)\end{array}$} \\
\cline { 2 - 3 } \cline { 5 - 6 } & $\mathrm{F}$ & $\mathrm{U}$ & & $\mathrm{F}$ & $\mathrm{U}$ \\
\hline$\overline{\mathrm{C}}$ & $9.33^{*}$ & .102 & & $27.84^{* *}$ & .045 \\
$\sigma$ & $63.10^{* *}$ & .691 & & $188.17^{* *}$ & .302 \\
$\mathrm{~W}_{\mathrm{I}}$ & $43.83^{* *}$ & .480 & & $142.28^{* *}$ & .228 \\
\hline
\end{tabular}

${ }^{*} p<.01 ;{ }^{* *} p<.001$. 
A particularly interesting feature of the individual differences analysis is the role of mean criterion level. In both regression equations, the sign of the weight for $\bar{C}$ is negative, indicating the predicted negative relation with ER. In both experiments, however, the correlation coefficient between the two variables is positive. In the deadline experiment, it is significantly so $\left(\mathrm{z} / \mathrm{SE}_{\mathrm{z}}=3.44, \mathrm{p}<.001\right)$. In that experiment, the correlation between ER and median RT of the correct response, .587 , is also significantly positive $\left(z / \mathrm{SE}_{\mathrm{z}}=2.76, \mathrm{p}<.01\right)$. This does not support the simple expectation of between-subjects tradeoff in which fast subjects with low criteria would make the most errors. This situation, which was previously observed by Grice et al. (1979), leads to a different, but informative, conclusion. The difference in direction of the relation between ER and $\overline{\mathrm{C}}$, indicated by the correlation and the regression weight, is somewhat clarified by the partial correlations. For the deadline experiment, the direct correlation is .625 ; the partial correlation with $\mathrm{W}_{\mathrm{I}}$ held constant is -.209 ; and the partial correlation with both $\mathrm{W}_{\mathrm{I}}$ and $\sigma$ held constant is -.750 . This last negative value is significant $\left(\mathrm{z} / \mathrm{SE}_{\mathrm{z}}=4.35, \mathrm{p}<.001\right)$. The picture is less dramatic in the accuracy experiment, but the partial correlation with both of the other variables held constant is - .587, and is significant $\left(\mathrm{z} / \mathrm{SE}_{\mathrm{z}}=2.78, \mathrm{p}<.01\right)$.

We believe that there is a rather simple explanation of this analysis. It appears that the major factors determining individual differences in error rate are criterion variability and the kind of short latency discriminative ability reflected by $\mathrm{W}_{\mathrm{I}}$. A subject deficient in one or both of these abilities must adopt a relatively high criterion and sacrifice speed in order to avoid an unacceptable error rate. As predicted, the effect of an elevated criterion is to reduce error rate, but only below a level determined by the other factors.

\section{DISCUSSION}

The theory, developed in the context of an accuracy experiment, not only provided a satisfactory description of the speeded experiment, but did so in terms of the single parameter, $\bar{C}$. This was the most simple and obvious prediction, and was confirmed by several aspects of the data. In terms of individual subject parameter estimates, $\bar{C}$ was the only one differing significantly between the experiments. For the group data, the prediction of parallel growth functions for both the correct and incorrect responses was confirmed. Finally, the single parameter solution provided good fits to both the correct and error RT distributions. The common VCDF, applicable to both experiments, was an implication of these findings. It should be noted, however, that the successful application of the theory did not depend upon an adequate single-parameter solution. The variables, $o$ and $W_{I}$, are also subject to sampling error and to possible influence by experimental manipulations.

Also, the theory resulted in satisfactory descriptions of both the correct and error RT distributions for the individual subjects of the deadline experiment. In doing so, it provided the measures for an analysis of individual differences in theoretical terms. At this time, such an analysis appears to be unique to this approach. One interesting result of analysis of the deadline experiment was that the combination of $\sigma$ and $\bar{C}$, which are estimated from the correct functions only, accounted for $74 \%$ of the variance in error rate. Another important finding was that, while the difference in error rate between experiments was due almost entirely to a difference in criterion level, differences among subjects within the experiments were primarily due to differences in criterion variability and inhibitory ability.

We believe that theory of this kind has considerable promise of providing a much more complete analysis of the effects of experimental variables on tradeoff than has been previously available. For example, Jennings, Wood, and Lawrence (1976) have presented a very interesting study of the effect of graded doses of alcohol on the speed-accuracy tradeoff. They wisely chose to study the tradeoff between conditions on the grounds that the SATF is less likely to be confounded with criterion effects than is the CAF. They concluded that alcohol does tend to lower the criterion, but also impares efficiency, indicated by decreasing the slope of the SATF. The authors pointed out that their results did not identify the specific functional process which was impaired. Use of a theory of the present type, in which the function parameters for their experimental situation had been determined, should provide considerable additional diagnostic power. Direct measures of criterion level and variability and of inhibitory ability are available. In addition to a simple SATF, it would be possible to present a family of VCDFs, one representing each condition. Divergence of these functions would indicate an effect upon either $\sigma$ or $W_{I}$, and more complete analysis would further localize the effect. ${ }^{5}$ While the associative factors $A$ and $A_{D}$ are invariant in the present application, that would not necessarily be so in the case of a potent experimental variable, and this possibility could also be investigated. This example is given as an illustration of the kind of analysis which the theory now makes possible with respect to any experimental or clinical variable affecting the speed-accuracy tradeoff.

\section{REFERENCES}

Anderson, N. H. Algebraic models in perception. In E. C. Carterette \& M. P. Friedman (Eds.), Handbook of perception (Vol. II). Psychophysical judgment and measurement. New York: Academic Press, 1974. 
Grice, G. R., Nullmeyer, R., \& Spiker, V. A. Application of variable criterion theory to choice reaction time. Perception \& Psychophysics, 1977, 22, 431-449.

Grice, G. R., Spiker, V. A., \& Nullmeyer, R. Variable criterion analysis of individual differences and stimulus similarity in choice reaction time. Perception \& Psychophysics, 1979, 25, 353-370.

Jennings, J. R., Wood, C. C., \& Lawrence, B. E. Effects of graded doses of alcohol on speed-accuracy tradeoff in choice reaction time. Perception \& Psychophysics, 1976, 19, 85-91.

Wood, C. C., \& Jennings, J. R. Speed-accuracy tradeoff functions in choice reaction time: Experimental designs and computational procedures. Perception \& Psychophysics, 1976, 19, 92-102.

\section{NOTES}

1. The terms SATF and CAF are attributed by Wood and Jennings to an unpublished paper by R.T. Ollman.

2. Graphs of the functions for $V, A, I$, and $A_{D}$ and a discussion of their origins and interpretations are given by Grice et al. (1979).

3. The steps in obtaining the predicted distributions are as follows:

(1) Obtain estimates of the cumulative probability of each response reaching threshold, $F_{c}(t)$ and $F_{e}(t)$, by applying Equations 8 and 9 to the data for each subject.

(2) Obtain estimates of the growth of $E_{c}(t)$ and $E_{e}(t)$ by transforming $F_{c}(t)$ and $F_{e}(t)$ to normal deviates. This was done at 20 -msec intervals.

(3) Obtain estimates of $\sigma$ and $\bar{C}$ from the slopes and intercepts, respectively, of the inverse of linear functions response evocation characteristics (RECs) relating the function for correct responses, $E_{c}(t)$, for each subject to that calculated from the theory at corresponding intervals of time. The theoretical function is the sum of Equations 3 and 4. In the present application, the subject scale values were weighted by the MüllerUrban weights in computing the least squares regression. All of the 25 functions were appropriately linear.

(4) Transform the theoretical functions, Equations 3-6, to the scales of the individual subject. Equation $3, \mathrm{~V}$, is transformed by the linear regression equations from Step 3. (It is not the inverse functions used for this purpose.) This sets the origin at the mean of the subject's criterion distribution. The remaining three functions are transformed by the slope parameter only.
(5) Obtain estimates of $W_{1}$. In previous applications, these estimates were based on a single point only, but here a somewhat improved method was used. First, from the transformed equations, we computed the values of $E_{e}(t)$ omitting $l(t)$, i.e., $E_{c}(t)-A_{D}(t)$. From Equation 2, then, an estimate of the "obtained" $I(t)$ is given by

$$
I(t) \text { (obtained) }=E_{c}(t)-A_{D}(t)-E_{e}(t) \text { obtained. }
$$

The value of $I(t)$ calculated from Equation 5 is based on an equal weight, $W_{I}=1$, for all subjects. Both the calculated and obtained values were calculated at 40 -msec intervals up to $400 \mathrm{msec}$. The value of $W_{1}$ was given by the slope of a least squares regression line, through the origin of the relation of $I(t)$ (obtained) to I(t) (calculated) at $40-\mathrm{msec}$ intervals. Early, unreliable scale values based on error rates of .01 and under were excluded unless no other data existed.

(6) The transformations of Equation $S$ are then weighted by the factor $W_{1}$.

(7) Obtain the predicted functions, $F_{c}(t)$ and $F_{e}(t)$, describing the theoretical, cumulative probability of each response reaching criterion. This is the area of the unit normal function below successive values of $E_{c}(t)$ and $E_{c}(t)$ computed from the fully transformed Equations 1 and 2.

(8) Obtain, by subtraction, the successive values of $\Delta F_{c}(t)$ and $\Delta F_{e}(t)$ for brief intervals. The interval used here for the individual subjects was $.04 \mathrm{sec}$.

(9) Obtain the predicted cumulative distributions of correct responses and errors by the successive application of Equations 7 and 8 , and summating the values of $\Delta P_{c}(t)$ and $\Delta \mathrm{P}_{\mathrm{e}}(\mathrm{t})$.

4. Estimates, presented by Grice et al. (1977), of the measures of skewness $\left(y_{1}\right)$ and kurtosis $\left(y_{2}\right)$ were computed. For the accuracy experiment, the estimate of $\gamma_{1}$ was -.018 and for the deadline experiment it was .031 . The estimates of $\gamma_{2}$ were .355 and .349 for accuracy and deadline, respectively. Ignoring the negligible skewness, this implies that both criterion distributions resembled the $t$ distributions with 21 degrees of freedom. This close approximation to normality implies that a scaling solution based on the normal model would not introduce discernible distortion.

5 . As indicated previously, with only a difference in criterion level, $\bar{C}$, a point on a VCDF is equal to $I(t)+A_{D}(t)$. However, if other parameters differ, the full value is $\left(W_{1} \times I+A_{D}\right) / o$.

(Received for publication March 19, 1979; revision accepted June 5, 1979.) 